Z Rheumatol 2021 $\cdot 80: 688-691$

https://doi.org/10.1007/s00393-021-01053-9

Angenommen: 29. Juni 2021

Online publiziert: 23. Juli 2021

(c) Der/die Autor(en) 2021

\section{Mustercurriculum der Deutschen Gesellschaft für Rheumatologie für die Weiterbildung im Fachgebiet Innere Medizin und Rheumatologie}

\author{
Alexander Pfeil' $\cdot$ Martin Krusche ${ }^{2}$. Diana Vossen ${ }^{3} \cdot$ Michael N. Berliner $^{4}$. \\ Gernot Keyßer ${ }^{5}$ Andreas Krause ${ }^{6} \cdot$ Hanns-Martin Lorenz ${ }^{7} \cdot$ Bernhard Manger ${ }^{8}$. \\ Florian Schuch ${ }^{9}$ Christof Specker ${ }^{10}$. Jürgen Wollenhaupt ${ }^{11} \cdot$ Xenofon Baraliakos $^{12}$. \\ Martin Fleck ${ }^{13,14}$. Fabian Proft ${ }^{15}$ \\ ' Klinik für Innere Medizin III, Funktionsbereich Rheumatologie, Universitätsklinikum Jena, Jena, \\ Deutschland; ${ }^{2}$ Medizinische Klinik mit Schwerpunkt Rheumatologie und Klinische Immunologie, Charite \\ Universitätsmedizin, Berlin, Deutschland; ${ }^{3}$ Rheinisches Rheumazentrum Meerbusch-Lank, St. Elisabeth \\ Hospital, Meerbusch, Deutschland; ${ }^{4}$ Rheumatologie und Geriatrie, Helios Klinikum Berlin-Buch, Berlin, \\ Deutschland; ${ }^{5}$ Department für Innere Medizin, Klinik für Innere Medizin II, Universitätsklinikum Halle, \\ Halle (Saale), Deutschland; ${ }^{6}$ Klinik für Innere Medizin, Abteilung Rheumatologie, klinische Immunologie \\ und Osteologie, Immanuel Krankenhaus Berlin, Berlin, Deutschland; ${ }^{7}$ Sektion Rheumatologie, \\ Medizinische Klinik V, Universitätsklinikum Heidelberg, Heidelberg, Deutschland; ${ }^{8}$ Medizinische Klinik 3, \\ Rheumatologie und Immunologie, Universitätsklinikum Erlangen, Friedrich-Alexander Universität \\ Erlangen-Nürnberg, Erlangen, Deutschland; ${ }^{9}$ Internistische Praxisgemeinschaft Rheumatologie - \\ Nephrologie, Erlangen, Deutschland; ${ }^{10} \mathrm{Klinik}$ für Rheumatologie und Klinische Immunologie, \\ Evangelisches Krankenhaus Kliniken Essen-Mitte, Essen, Deutschland; "Rheumatologie im \\ Struenseehaus, Hamburg, Deutschland; ${ }^{12}$ Rheumazentrum Ruhrgebiet, Ruhr-Universität Bochum, Herne, \\ Deutschland; ${ }^{13}$ Klinik und Poliklinik für Innere Medizin I, Universitätsklinikum Regensburg, Regenburg, \\ Deutschland; ${ }^{14}$ Klinik und Poliklinik für Rheumatologie/Klinische Immunologie, Asklepios Klinikum Bad \\ Abbach, Bad Abbach, Deutschland; ${ }^{15}$ Abteilung für Rheumatologie, Medizinische Klinik für \\ Gastroenterologie, Infektiologie und Rheumatologie, Campus Benjamin Franklin, Charité \\ Universitätsmedizin, Berlin, Deutschland
}

Alle Autoren schreiben im Auftrag der Kommission Fort- und Weiterbildung der Deutschen Gesellschaft für Rheumatologie.

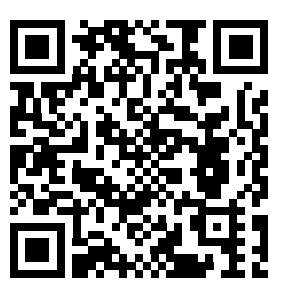

QR-Code scannen \& Beitrag online lesen

\section{Einleitung}

Mit der Implementierung der Musterweiterbildungsordnung (MWBO) 2018 erfolgten die Anpassung der Gebietsdefinition sowie die Neustrukturierung der Weiterbildungszeit für den Facharzt bzw. die Fachärztin für Innere Medizin und Rheumatologie [2].

Die Mindestweiterbildungszeit umfasst hierfür 72 Monate. Hierbei wird die Weiterbildungszeit in 2 Teile zu je 36 Monaten aufgeteilt, welche auf der einen Seite die gemeinsamen Inhalte der Facharztweiterbildungen im Gebiet der Inneren Medizin („Basisweiterbildung“) als auch die spezifischen Inhalte der Facharztweiterbildung Innere Medizin und Rheumatologie umfasst.

Im allgemeinen Teil "Innere Medizin" erfolgt der Erwerb von mindestens 2 Fach- arztkompetenzen, die nicht dem Schwerpunkt angehören, über 24 Monate sowie jeweils eine 6-monatige Tätigkeit in einer Notaufnahme und der Intensivmedizin. Die spezialisierte Weiterbildungszeit im Fachgebiet Innere Medizin und Rheumatologie erstreckt sich ebenfalls über 36 Monate, von denen nach der MWBO mindestens 24 Monate in der stationären rheumatologischen Versorgung vorgesehen sind [2]. Von dieser Aufteilung abweichend, wurden in einzelnen Landesärztekammern Änderungen vorgenommen, die eine längere Weiterbildungszeit in der ambulanten rheumatologischen Versorgung ermöglichen.

Basierend auf der MWBO von 2018, wurde von der Kommission für Fort- und Weiterbildung der Deutschen Gesellschaft für Rheumatologie (DGRh) im Auftrag des Vorstandes der DGRh ein Mustercurricu- 
lum hinsichtlich der Kernkompetenzen in der Weiterbildung im Fachgebiet Innere Medizin und Rheumatologie entwickelt. Dieses Mustercurriculum fokussiert auf die Weiterbildungskompetenzen im Schwerpunkt während der 36 Monate Weiterbildungszeit im Fachgebiet Innere Medizin und Rheumatologie und beinhaltet keine Weiterbildungskompetenzen für den allgemeinen Teil der Inneren Medizin („Basisweiterbildung").

Im Rahmen dieses Übersichtsartikels soll das Mustercurriculum der Deutschen Gesellschaft für Rheumatologie für die Weiterbildung im Fachgebiet Innere Medizin und Rheumatologie vorgestellt werden.

\section{Die Musterweiterbildungsordnung (MWBO)}

In der MWBO 2018 werden „kognitive und Methodenkompetenzen" (Kenntnisse) und "Handlungskompetenzen" (Erfahrungen und Fertigkeiten) für das Fachgebiet Innere Medizin und Rheumatologie vermittelt [1, 2], welche die Erkennung, Diagnosestellung, Differenzialdiagnose, Therapie, Langzeitbetreuung und Rehabilitation entzündlich-rheumatischer Gelenkerkrankungen, inflammatorischer bzw. immunologischer Systemerkrankungen (insbesondere Kollagenosen und Vaskulitiden), autoinflammatorischer Syndrome, Immundefekte und der Komorbiditäten beinhalten [2]. Angelehnt an die MWBO 2018 erfolgte die Entwicklung des Mustercurriculums durch die Kommission Fort- und Weiterbildung der Deutschen Gesellschaft für Rheumatologie.

\section{Das Mustercurriculum}

Ziel des Mustercurriculums ist die Etablierung eines Leitfadens für Weiterbilderinnen und Weiterbilder sowie Weiterzubildende in der Rheumatologie, in dem die Weiterbildungskompetenzen der 36-monatigen Weiterbildungszeit im Fachgebiet Innere Medizin und Rheumatologie, basie-

Abkürzungen

$\begin{array}{ll}\text { DGRh } & \begin{array}{l}\text { Deutsche Gesellschaft für Rheuma- } \\ \text { tologie }\end{array} \\ \text { MWBO } & \text { Musterweiterbildungsordnung }\end{array}$

rend auf der MWBO 2018, strukturiert und zeitlich abgestimmt vermittelt werden [2]. Das Mustercurriculum soll auch als Grundlage für alle Weiterbilderinnen und Weiterbilder bei der Ausarbeitung eines eigenen Weiterbildungsplanes dienen, der mit der Umsetzung der MWBO bei den Landesärztekammern bei der erforderlichen Neubeantragung der Weiterbildungsbefugnis eingereicht werden muss.

Als Grundlage für das Mustercurriculum dienen die in der MWBO 2018 aufgeführten definierten Untersuchungsund Behandlungsverfahren [2]. Ziel des Mustercurriculums ist das strukturierte Erlernen der körperlichen Untersuchung, Diagnose, Differenzialdiagnose, Therapiealgorithmen, Langzeitbetreuung und Rehabilitation entzündlich-rheumatischer Gelenkerkrankungen, inflammatorischer bzw. immunologischer Systemerkrankungen (insbesondere Kollagenosen, Vaskulitiden und autoinflammatorische Syndrome) sowie deren assoziierten Komorbiditäten.

\section{Aufbau des Mustercurriculums}

Die zu vermittelnden und zu erlernenden Kompetenzen wurden auf 3 Weiterbildungsjahre aufgeteilt. Bei den Kompetenzen handelt es sich um Mindestanforderungen, welche in Adaptation an die jeweilige Weiterbildungsstätte angepasst werden sollten, sodass entsprechende Tätigkeiten oder Kenntnisse zu einem früheren (aber auch späteren) Zeitpunkt erlernt oder durchgeführt werden können. Selbstverständlich ist eine vertiefte Kompetenzvermittlung über das vorliegende Mustercurriculum hinaus möglich und wünschenswert und kann entsprechend im individuellen Weiterbildungsplan der Weiterbildungsstätte aufgenommen werden. Die hier vorgenommene Strukturierung der Weiterbildungskompetenzen wurde in der Kommission für Fort- und Weiterbildung erarbeitet und vom Vorstand der DGRh konsentiert und soll einen zeitlich abgestimmten Kompetenzerwerb ermöglichen. Additiv werden variable Weiterbildungsinhalte abgebildet, welche keine Zuordnung zum Weiterbildungsabschnitt erfahren und entsprechend dem Kenntnisstand im ersten bis dritten Weiterbildungsjahr vermittelt werden können.
Der Kompetenzerwerb folgt einer Gliederung des Curriculums hinsichtlich des Erlernens von Leitsymptomen, Untersuchung, Labor, Radiologie, rheumatologischer Diagnostik, physikalischer Therapie und therapeutischen Maßnahmen. Zusätzlich erfolgt im zweiten und dritten Weiterbildungsjahr das Erlernen von Interventionen (z.B. Gelenkpunktion) (Details S. - Tab. 1).

Als variable Inhalte, welche keinem Weiterbildungsabschnitt zugeordnet werden, sind die Osteologie, Schulungsprogramme und Themen des Strahlenschutzes zu nennen (s. • Tab. 2).

\section{Anpassung des Mustercurriculums}

Das Mustercurriculum reflektiert die Mindestanforderungen der MWBO. In diesem Zusammenhang ist das Mustercurriculum nicht als optimale und bindende Weiterbildungsvariante für jede rheumatologische Weiterbildungsstätte in Deutschland anzusehen, sondern gibt den Rahmen für eine erfolgreiche Weiterbildung zum/zur Facharzt/Fachärztin für Innere Medizin und Rheumatologie vor. Eine Anpassung des Curriculums an die jeweiligen Gegebenheiten des Weiterbildungsstandortes ist jederzeit möglich bzw. ggf. notwendig. Dies beinhaltet Änderungen der zeitlichen Abfolge der Wissensvermittlung, eine vertiefte Weiterbildung in einzelnen Aspekten sowie die Hinzunahme von weiteren Weiterbildungsinhalten.

Für eine kontinuierliche Weiterentwicklung des Curriculums benötigt die Kommission für Fort- und Weiterbildung eine Rückmeldung der Weiterbilderinnen und Weiterbilder sowie der Weiterzubildenden hinsichtlich der Weiterbildungsinhalte, -themen und Struktur des Curriculums. Somit ist das Curriculum als "Work in Progress" zu sehen, weshalb ausdrücklich Kommentare oder auch Verbesserungsvorschläge an die Kommission erwünscht sind.

Zusammenfassend soll durch das Mustercurriculum eine standardisierte und strukturierte Aus- und Weiterbildung von Fachärztinnen und Fachärzten für Innere Medizin und Rheumatologie gewährleistet werden. 


\section{Empfehlungen und Stellungnahmen von Fachgesellschaften}

Tab. 1 Mindestanforderungen an den Kompetenzerwerb, wobei die Tätigkeiten und Kenntnisse zu einem früheren oder späteren Zeitpunkt als angegeben erlernt bzw. durchgeführt werden können

Kompetenzerwerb

1. Jahr Leitsymptom: Erlernen einer gezielten rheumatologischen Anamnese mit Fokussierung auf das führende Leitsymptom und hierfür möglicherweise zugrunde liegende entzündlich-rheumatische Erkrankungen

Untersuchung: Erlernen einer gezielten rheumatologischen körperlichen Untersuchung inklusive möglicher systemischer Beteiligungen entzündlich-rheumatischer Erkrankungen

Labor: Erlernen einer gezielten Indikationsstellung sowie Einführung in Methodik, Durchführung und Einordnung von Laboruntersuchungen sowie immunologischer Parameter in die entzündlich-rheumatischen Krankheitsbilder

Radiologie: Erlernen der gezielten Indikationsstellung radiologischer Untersuchungen sowie die Einordnung der Befunde in die entzündlich-rheumatischen Krankheitsbilder

Rheumatologische Diagnostik: Einführung in gezielte rheumatologisch-immunologische Diagnostik mittels Arthro- und Gefäßsonographie, Kapillarmikroskopie, Analyse der Synovialflüssigkeit sowie rheumatologische/immunologische Labordiagnostik einschließlich Autoantikörper und immungenetische Testung unter engmaschiger Anleitung

Physikalische Therapie: Kenntnisse physikalischer, krankengymnastischer und ergotherapeutischer Behandlungsprinzipien spezifischer entzündlich-rheumatischer Erkrankungen und degenerativer Gelenkerkrankungen sowie Wirbelsäulenerkrankungen

Therapie:

- Kenntnisse über die Erstellung eines medikamentösen Therapieplans zur Behandlung entzündlich-rheumatischer Erkrankungen und degenerativer Gelenkerkrankungen

- Erlernen des Erkennens von - und den Umgang mit - möglichen therapieassoziierten unerwünschten Ereignissen

- Selbstständige Verordnung medikamentöser Therapien sowie von Hilfsmitteln anhand der Heilmittelverordnung zur Behandlung entzündlich-rheumatischer Erkrankungen und degenerativer Gelenkerkrankungen

- Erlernen der interdisziplinären Indikationsstellung zu chirurgischen, strahlentherapeutischen und nuklearmedizinischen Behandlungsverfahren

- Mitbetreuung von Patienten mit entzündlich-rheumatischen Erkrankungen und degenerativen Gelenkerkrankungen unter Supervision

- Erstellen strukturierter rheumatologischer Befunde/Arztbriefe unter Supervision

2. Jahr Leitsymptom/Diagnose/Differenzialdiagnose: Vertiefung der gezielten rheumatologischen Anamnese zur differenzialdiagnostischen Einordnung der geschilderten Beschwerdesymptomatik

Untersuchung:Vertiefung der gezielten rheumatologischen körperlichen Untersuchung inklusive möglicher systemischer Beteiligungen entzündlich-rheumatischer Erkrankungen

Selbstständige Betreuung von Patienten mit entzündlich-rheumatischen Erkrankungen und degenerativen Gelenkerkrankungen unter Supervision

Radiologie: vertiefende Kenntnisse der Auswertung radiologischer Verfahren zur Diagnostik entzündlich-rheumatischer und degenerativer Gelenkerkrankungen sowie möglicher therapieassoziierter unerwünschter Ereignisse und die differenzialdiagnostische Einordnung dieser Befunde in die entzündlich-rheumatischen Krankheitsbilder

Rheumatologische Diagnostik: selbstständige Durchführung gezielter rheumatologisch-immunologischer Diagnostik mittels Arthrosonographie, Gefäßsonographie, Kapillarmikroskopie, Analyse der Synovialflüssigkeit sowie rheumatologische/immunologische Labordiagnostik einschließlich Autoantikörper, zelluläre Immunität und immungenetische Testung unter Supervision

Interventionen: Einführung in invasive diagnostische und therapeutische Verfahren (wie z. B. Gelenkpunktion, Injektionstherapien und/ oder Lippendrüsen- und Hautbiopsien) unter engmaschiger Anleitung

Therapie: selbstständige Erstellung eines medikamentösen Therapieplans zur Behandlung entzündlich-rheumatischer Erkrankungen und degenerativer Gelenkerkrankungen inklusive Aufklärung der Patientinnen/Patienten unter Supervision

3. Jahr Anamnese, Diagnostik, Therapie: selbstständige Betreuung von Patienten mit entzündlich-rheumatischen Erkrankungen und degenerativen Gelenkerkrankungen mit der Möglichkeit zur Rücksprache bei Bedarf

Erwerb von Grundkenntnissen in physikalischer und rehabilitativer Medizin sowie zu Fragestellungen der rheumatologischen Begutachtung

Erwerb von Kenntnissen zur Transition von Patienten mit pädiatrisch-rheumatologischen Krankheitsbildern

Erwerb von Grundkenntnissen zur Planung und Betreuung von Schwangerschaften bei Patientinnen mit entzündlich-rheumatischen Erkrankungen

Erwerb von Grundkenntnissen zur Diagnostik und Therapie von primären und sekundären Immundefekten

Radiologie: Auswertung radiologischer Verfahren zur Diagnostik entzündlich-rheumatischer und degenerativer Gelenkerkrankungen sowie möglicher therapieassoziierter unerwünschter Ereignisse und die differenzialdiagnostische Einordnung dieser Befunde in die entzündlich-rheumatischen Krankheitsbilder

Rheumatologische Diagnostik: selbstständige Durchführung gezielter rheumatologisch immunologischer Diagnostik mittels Arthrosonographie, Gefäßsonographie (inklusive Duplexsonographie zur Akutdiagnostik der Vaskulitiden), Ultraschall der Speicheldrüsen, Kapillarmikroskopie, Analyse der Synovialflüssigkeit sowie rheumatologische/immunologische Labordiagnostik einschließlich Autoantikörper und immungenetische Testung mit der Möglichkeit zur Rücksprache bei Bedarf sowie Erlernen der Qualitätssicherung gemäß RiLiBÄK-Richtlinien

Interventionen: Durchführung invasiver diagnostischer und therapeutischer Verfahren (wie z. B. Gelenkpunktion und Injektionstherapien und anderen) unter Supervision 
Tab. 2 Weiterbildungsinhalte mit einer variablen Zuordnung zum Weiterbildungsabschnitt

\begin{tabular}{|c|c|}
\hline & Kompetenzerwerb \\
\hline \multirow[t]{3}{*}{$\begin{array}{l}\text { Zeitlich vari- } \\
\text { able Zuord- } \\
\text { nung }\end{array}$} & $\begin{array}{l}\text { Osteologie: } \\
\text { - Diagnostik und konservative Therapie der Osteoporose und Osteomalazie } \\
\text { bzw. osteologischer Erkrankungsbilder } \\
\text { - Indikationsstellung, Durchführung und Auswertung der DXA-Knochenmine- } \\
\text { raldichtemessung }\end{array}$ \\
\hline & $\begin{array}{l}\text { Schulungsprogramme: Kennenlernen und Erlernen von strukturierten Pa- } \\
\text { tientenschulungsprogrammen bei rheumatischen und muskuloskeletalen } \\
\text { Erkrankungen }\end{array}$ \\
\hline & $\begin{array}{l}\text { Strahlenschutz: } \\
\text { - Grundlagen der Strahlenbiologie und Strahlenphysik bei der Anwendung } \\
\text { ionisierender Strahlen am Menschen } \\
\text { - Grundlagen des Strahlenschutzes beim Patienten und Personal einschließ- } \\
\text { lich der Personalüberwachung und des baulichen und apparativen Strahlen- } \\
\text { schutzes }\end{array}$ \\
\hline
\end{tabular}

\section{Fazit für die Praxis}

- Das Mustercurriculum ermöglicht die standardisierte Vermittlung von Kernkompetenzen im Rahmen der Facharztweiterbildung für Innere Medizin und Rheumatologie.

- Die zu erlernenden Kernkompetenzen und die variabel zuzuordnenden Weiterbildungsinhalte werden in 3 Ausbildungsabschnitten über 36 Monate vermittelt.

- Eine Anpassung des Curriculums an die Gegebenheiten des jeweiligen Weiterbildungsortes ist sinnvoll bzw. notwendig.

Schlüsselwörter. Musterweiterbildungsordnung $\cdot$ Kernkompetenzen · Facharztweiterbildung · Ausbildungsabschnitte - Weiterbildungsort

\section{Korrespondenzadresse}

\section{PD Dr. med. habil. Alexander Pfeil}

Klinik für Innere Medizin III, Funktionsbereich Rheumatologie, Universitätsklinikum Jena Am Klinikum 1, 07747 Jena, Deutschland alexander.pfeil@med.uni-jena.de

Funding. Open Access funding enabled and organized by Projekt DEAL.

\section{Einhaltung ethischer Richtlinien}

Interessenkonflikt. A. Pfeil, M. Krusche, D. Vossen, M.N. Berliner, G. Keyßer, A. Krause, H.-M.Lorenz, B. Manger, F. Schuch, C. Specker, J. Wollenhaupt, X. Baraliakos, $M$. Fleck und F. Proft geben an, dass kein Interessenkonflikt besteht.

Für diesen Beitrag wurden von den Autoren keine Studien an Menschen oder Tieren durchgeführt. Für die aufgeführten Studien gelten die jeweils dort angegebenen ethischen Richtlinien.

Open Access. Dieser Artikel wird unter der Creative Commons Namensnennung 4.0 International Lizenz veröffentlicht, welche die Nutzung, Vervielfältigung, Bearbeitung, Verbreitung und Wiedergabe in jeglichem Medium und Format erlaubt, sofern Sie den/die ursprünglichen Autor(en) und die Quelle ordnungsgemäß nennen, einen Link zur Creative Commons Lizenz beifügen und angeben, ob Änderungen vorgenommen wurden.

Die in diesem Artikel enthaltenen Bilder und sonstiges Drittmaterial unterliegen ebenfalls der genannten Creative Commons Lizenz, sofern sich aus der Abbildungslegende nichts anderes ergibt. Sofern das betreffende Material nicht unter der genannten Creative Commons Lizenz steht und die betreffende Handlung nicht nach gesetzlichen Vorschriften erlaubt ist, ist für die oben aufgeführten Weiterverwendungen des $\mathrm{Ma}$ terials die Einwilligung des jeweiligen Rechteinhabers einzuholen.

Weitere Details zur Lizenz entnehmen Sie bitte der Lizenzinformation auf http://creativecommons.org/ licenses/by/4.0/deed.de.

\section{Literatur}

1. Fleck M, Berliner MN, Krause A (2021) Novel revision of the training regulations for German physicians: consequences for trainees and trainers in rheumatology. ZRheumatol 80:9-13

2. Bundesärztekammer (2018) (Muster-)Weiterbildungsordnung 2018. https://www. bundesaerztekammer.de/aerzte/aus-weiterfortbildung/weiterbildung/muster-weiter bildungsordnung/.Zugegriffen:20.07.2021.
Machen Sie sich fit mit dem „Facharzt-Training Innere Medizin“!

Bereiten Sie sich auf die Facharztprüfung vor oder möchten Sie Ihr fachspezisches Wissen mit typischen Fallbeispielen aus der Inneren Medizin auffrischen? Dann sind die Sonderhefte von Der Internist "FacharztTraining Innere Medizin" genau das Richtige für Sie.

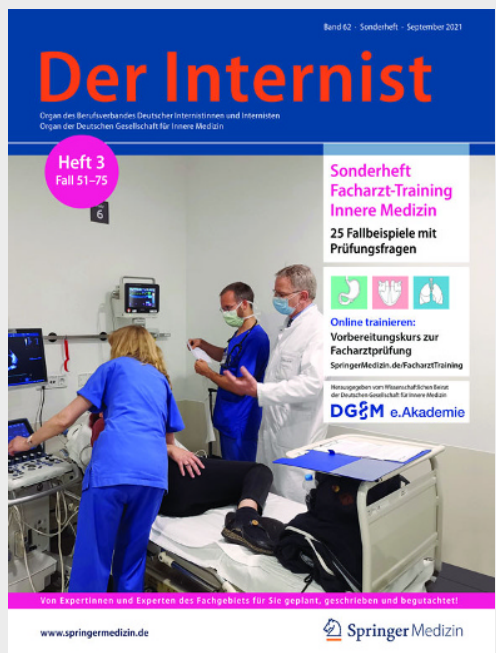

Sie finden in diesen Heften:

- Typische, alltagsnahe Fallbeispiele, systematisch und aktuell aufbereitet mit Prüfungsfragen und deren Antworten

- Kompaktes Wissen aus allen Fachbereichen der Inneren Medizin

- Sonderheft 1, 2 und 3 sind erhältlich, weitere Sonderhefte folgen in Kürze

- Von Expertinnen und Experten für Sie geplant, geschrieben und begutachtet

- Herausgegeben vom wissenschaftlichen Beirat der Deutschen Gesellschaft für Innere Medizin

Bestellen Sie die Sonderhefte „FacharztTraining Innere Medizin" unter dem Aktionscode C0019640

bei Marie-Luise.Witschel@springer.com. (1 Sonderheft 44 EUR, Paketpreis 2 Sonderhefte 75 EUR, Paketpreis 3 Sonderhefte 99 EUR) 